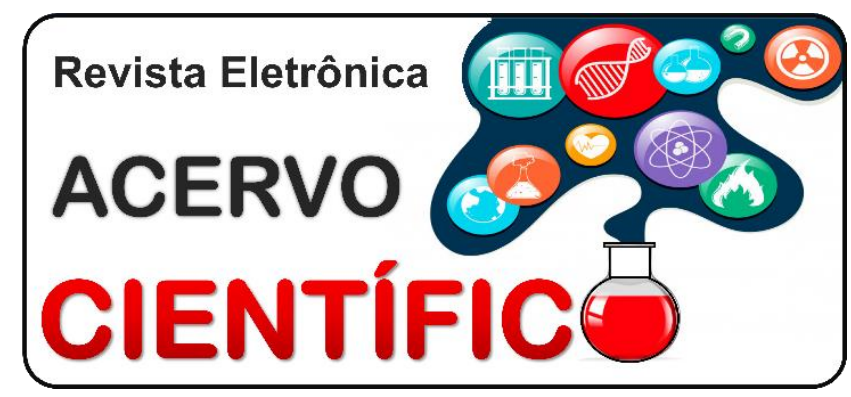

ARTIGO ORIGINAL

Recebido em: 8/2020

Aceito em: 8/2020

Publicado em: 11/2020

\title{
Tipos histológicos do câncer do colo do útero associado com a infecção pelo HPV em pacientes atendidas em hospital de referência oncológica no estado do Pará
}

\author{
Histological types of cervical câncer associated with HPV infection in patients attended in \\ a oncology referral Hospital in the State of Pará
}

Tipos histológicos de câncer cervical asociado a infección por VPH en pacientes atendidos en un hospital de referencia de oncología en el estado de Pará

Nathalia Moura Soares ${ }^{1 *}$, Manuelle França Teran Nunes de Paula ${ }^{1}$, Katharine Klicie Barros da Silva ${ }^{1}$, Gabrielly Carolline Machado Chaves ${ }^{1}$, Milena Cristo Mendonça ${ }^{1}$, Victória Moraes Perinazzo ${ }^{1}$, Carolle Freitas Batalha1, Thaiz Taiana Cardoso da Silva ${ }^{1}$, Allan Jhones Costa Gomes ${ }^{1}$, Cláudia Nazaré de Souza Almeida Titan Martins'.

\begin{abstract}
Resumo: Avaliar a prevalência dos tipos histológicos do câncer do colo do útero associado a infecção do HPV em pacientes atendidas em um hospital de referência oncológica em um município do estado do Pará. Estudo observacional e retrospectivo do tipo transversal de caráter descritivo, sendo pesquisa documental, baseado no levantamento de dados epidemiológicos secundários extraídos das fichas de tumor, e dos prontuários dos pacientes do Hospital escolhido. Total de 276 casos registrados oficialmente, observou-se a variável faixa etária a maior taxa correspondia ao grupo de 40 a 60 anos (202 registros - 73,18\%), sendo o grupo de 60 anos ou mais com 74 casos. No que consiste em relação aos fatores de riscos mais prevalentes em relação ao câncer de colo de útero, observou-se aqueles que possuíam os fatores de risco para câncer de colo de útero com a presença de HPV correspondiam à metade dos casos (com 138 casos - 50,0 \%), sendo que aqueles que não possuíam fatores de risco equivaleram a 105 casos $(38,0 \%)$, sendo aqueles sem informação com 33 casos (12,0\%). Conclui-se que há fatores que influenciam o diagnóstico tardio e a negligência de sinais e sintomas preditivos de câncer de colo de útero.
\end{abstract}

Palavras-chave: Neoplasias, Neoplasias do colo do útero, Epidemiologia.

\begin{abstract}
To evaluate the prevalence of histological types of cervical cancer associated with HPV infection in patients seen at an oncology referral hospital in a municipality in the state of Pará. Observational and retrospective cross-sectional study of a descriptive character, being documentary research, based on the survey of epidemiological data secondary data extracted from the tumor files, and from the patient records of the chosen Hospital. A total of 276 officially registered cases, the variable age group was observed, with the highest rate corresponding to the group aged 40 to 60 years (202 records - 73.18\%), with the group aged 60 or over having 74 cases. With regard to the most prevalent risk factors in relation to cervical cancer, it was observed that those who had the risk factors for cervical cancer with the presence of HPV corresponded to half of the cases (with 138 cases - 50.0\%), with those who did not have risk factors equivalent to 105 cases (38.0\%), with those without information having 33 cases $(12.0 \%)$. It is concluded that there are factors that influence the late diagnosis and the neglect of predictive signs and symptoms of cervical cancer.
\end{abstract}

Keywords: Neoplasms, Uterine cervical neoplasms, Epidemiology.

\footnotetext{
${ }^{1}$ Centro Universitário Metropolitano da Amazônia (UNIFAMAZ), Belém - Pará.

*E-mail: nathmouram594@gmail.com
} 
Resumen: Evaluar la prevalencia de los tipos histológicos de cáncer de cuello uterino asociados con la infección por VPH en pacientes atendidos en un hospital de referencia de oncología de un municipio del estado de Pará. Estudio transversal observacional y retrospectivo de carácter descriptivo, siendo investigación documental, basada en la encuesta de datos epidemiológicos. datos secundarios extraídos de los archivos del tumor y de los registros de pacientes del hospital elegido. En un total de 276 casos registrados oficialmente, se observó el grupo de edad variable, con la tasa más alta correspondiente al grupo de 40 a 60 años (202 registros - 73,18\%), con el grupo de 60 años o más con 74 casos. Con respecto a los factores de riesgo más prevalentes en relación con el cáncer de cuello uterino, se observó que aquellos que tenían los factores de riesgo de cáncer de cuello uterino con presencia de VPH correspondían a la mitad de los casos (con 138 casos - 50.0\%), con aquellos que no tenían factores de riesgo equivalentes a 105 casos (38.0\%), con aquellos sin información con 33 casos (12.0\%). Se concluye que hay factores que influyen en el diagnóstico tardío y el descuido de los signos y síntomas predictivos del cáncer cervical.

Palabras clave: Neoplasias, Neoplasias del cuello uterino, Epidemiologia.

\section{INTRODUÇÃO}

O câncer do colo do útero ou cancro cervical consiste em lesões intrauterinas, devido ao crescimento anormal e, desordenado das células cancerosas. A evolução desta afecção se dá, na maioria dos casos, de forma lenta, com duração média de 10 a 20 anos, passando por fases pré-clínicas detectáveis e curáveis com transformações intraepiteliais progressivas que podem evoluir para um carcinoma invasor (CASARIN MR, et al., 2011). Nesta fase os principais sintomas são: sangramento vaginal, corrimento e a transmissão ocorre pelo contanto sexual (SOUSA ACO, et al., 2018).

Este tipo de câncer tem como causa principal a infecção pelo Papiloma vírus Humano (HPV), e segundo a International Agency for Rearsech on Câncer (IARC) os subtipos 16 e 18 do vírus são mais prevalentes em mulheres com carcinoma no colo do útero, associado a 70\% destes cânceres (INCA, 2015). A mais eficaz estratégia para prevenção do câncer é o exame de colpocitologia oncótica, o qual estuda as células cervicais descamadas, e segundo o Sistema de Bethesda em 2014, a nomenclatura a ser utilizada nos laudos, constituem as lesões iniciais intraepiteliais escamosas de baixo grau (LSIL) e lesões intraepiteliais escamosas de alto-grau (HSIL) (INCA, 2016).

O HPV está presente em quase $100 \%$ dos casos de câncer do colo do útero sendo que, sua incidência nos países em desenvolvimento é cerca de cinco vezes maior do que em países mais ricos (FEBRASGO, 2017). Aproximadamente, $20 \%$ dos indivíduos sadios, em todo o mundo, estão infectados pelo HPV e a maioria desses com infecções assintomáticas e transitórias (INCA, 2015). No Brasil, o câncer do colo do útero associado ao HPV é o segundo tipo mais frequente entre as mulheres por meio do desenvolvimento das lesões precursoras não identificadas e tratadas e, por conseguinte, resulta em um problema de saúde pública (INCA, 2016).

O objetivo do presente artigo foi avaliar a prevalência dos tipos histológicos do câncer do colo do útero associado a infecção do HPV em pacientes atendidas em um hospital de referência oncológica de um município do estado do Pará no período de 2011 a 2013.

\section{MÉTODOS}

Trata-se de um estudo observacional e retrospectivo do tipo transversal de caráter descritivo, sendo pesquisa documental, recorrendo-se a métodos e técnicas adequados para a apreensão, compreensão e análise do material selecionado: a) textos oficiais do Ministério da Saúde (normas, portarias), sistematizados em uma Matriz Interpretativa, b) teses e artigos científicos relacionados ao tema da tese, cujos autores são reconhecidos no meio acadêmico da saúde coletiva e c) baseado no levantamento de dados extraídos dos prontuários dos pacientes.

Foi utilizada para avaliação dos dados uma amostra de 100 prontuários de pacientes cadastrados em um hospital de um município do estado do Pará, com o diagnóstico do câncer do colo de útero associado a 
infecção do HPV, no o período de janeiro de 2011 a dezembro de 2013. O projeto foi aprovado pelo Comitê de Ética em Pesquisa (CEP) sob número de parecer 1.963.978.

Foram avaliados os dados iniciais de 100 prontuários de pacientes do gênero feminino com idade entre 25 a 80 anos sobre os tipos histológicos do câncer de colo de útero com diagnóstico positivo para a infecção pelo HPV atendidas no hospital de referência oncológica no Estado do Pará durante o período de janeiro de 2011 a dezembro de 2013.

Quanto aos critérios de inclusão foram mulheres com idade entre 25 a 80 anos que tiveram diagnostico positivo para o câncer do colo do útero associado a infecção pelo HPV no período de janeiro de 2011 a janeiro 2013 em um hospital de um município do estado do Pará. Já os Critérios de exclusão foram prontuários de pacientes com idade inferior a 25 anos e superior a 80 anos; prontuários com dados incompletos; prontuários de pacientes diagnosticados apenas por exame e imagem; prontuários que tem ausência de HPV e os relacionados a outras causas; prontuários de pacientes procedentes de outros municípios do Estado do Pará; prontuários de pacientes sem biópsia.

Os dados foram coletados a partir do Registro Hospitalar de Câncer (RHC) e da Divisão de Arquivo Médico e Estatístico (DAME) de um hospital de um município do estado do Pará. A coleta de dados foi realizada por meio da análise das fichas contendo as principais variáveis epidemiológicas, tais como: idade, ocupação, escolaridade, etnia, estado conjugal, uso de tabagismo e uso de álcool. Além das variáveis epidemiológicas foram coletados também dados clínicos e de diagnósticos (estadiamento (TMN) e resultados de biopsia e sorologia para HPV) que servem para identificar os tipos histológicos e aqueles que estão associados infecção por HPV.

\section{RESULTADOS}

Pela análise, do total de 276 casos registrados oficialmente, observou-se que em relação a variável faixa etária a maior taxa correspondia ao grupo de 40 a 60 anos (202 registros - 73,18\%), sendo o grupo de 60 anos ou mais com 74 casos, aproximadamente $26,8 \%$. Na variável Raça, o grupo de maior taxa foi a Parda (com 163 casos $-59,1 \%$ ), seguido da Preta (21 casos $-7,6 \%$ ), Branca (18 registros $-6,5 \%$ ), Amarela (5 casos - 1,8 \%) e Indígena ( 2 casos - 0,7\%); vale ressaltar que cerca de $24,3 \%$ (67 casos) foram registrados como 'sem informação'. No quesito de análise do nível de escolaridade, a maior concentração foi observada no grupo de 'Fundamental Incompleto', com 32,61 \% dos registrados (90 casos), seguido do fundamental completo (52 casos - 18,84\%), Analfabetos ( 45 casos - 16,30\%), Nível médio (42 casos - 15,22\%), nível superior completo ( 12 casos $-4,34 \%$ ), respectivamente. Sendo que o grupo registrado como 'sem informação' abrangeu 12,68\% (35 casos). No que consiste a situação ocupacional a maior taxa correspondeu a 'trabalhadores sem ocupação', com 60 casos $(21,7 \%)$, sendo seguido do grupo de 'trabalhadoras assalariadas' (42 registros - 15,2\%), trabalhadoras informais (31 casos - 11,2\%), e outros grupos (trabalhadoras de nível médio; trabalhadoras de nível superior, empresários e produtores e promotores de venda) com a somatória de 17 registros, sendo $6,3 \%$.

Tabela 1 - Relação escolaridade e a presença de HPV.

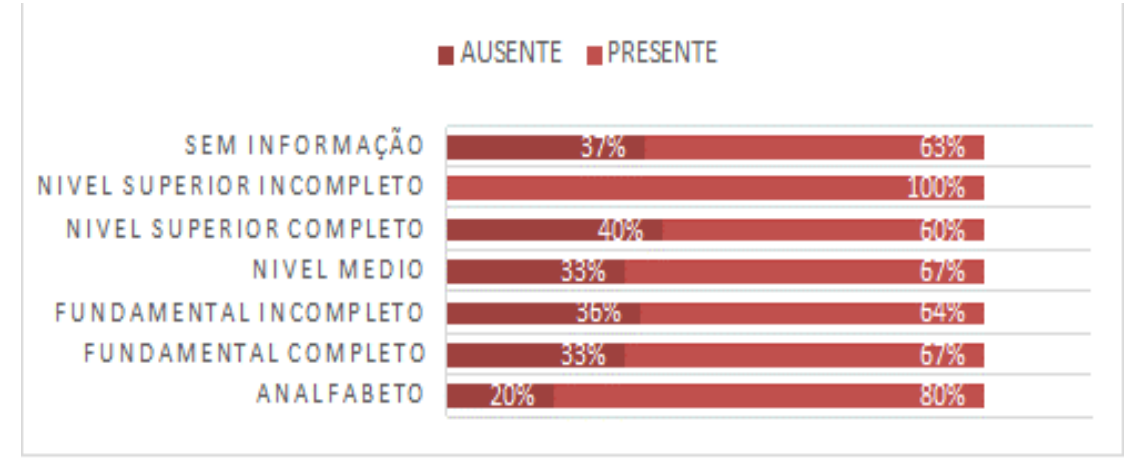

Fonte: SOARES NM, et al., 2020. 
No que consiste em relação aos fatores de riscos mais prevalentes em relação ao câncer de colo de útero, observou-se aqueles que possuíam os fatores de risco para câncer de colo de útero com a presença de HPV correspondiam à metade dos casos (com 138 casos - 50,0 \%), sendo que aqueles que não possuíam fatores de risco equivaleram a 105 casos (38,0\%), sendo aqueles sem informação com 33 casos (12,0\%). Em relação aos fatores de risco, a variável relacionada ao Álcool a maior taxa pertencia ao grupo 'ex-consumidor' com cerca de $56,5 \%$ do total (com 156 casos), seguido do grupo que 'nunca' realizou uso (84 casos - 30,4\%) e aqueles os quais 'sim' fizeram uso, com $6,9 \%$ dos casos (19 casos registrados). Em relação a Variável Tabaco, a maior taxa correspondeu ao grupo do 'ex-consumidor' (136 casos - 49,3\%), sendo seguido do grupo daqueles que 'nunca' fizeram uso (102 casos - 37,0\%), sendo apenas 18 casos do' grupo que 'sim' fez uso da substância (6,5\%). Em relação ao que consiste a análise do Estado Conjugal dos prontuários, registouse a maior taxa no grupo de 'Solteiro' (87 casos - 31,5\%), seguido de 'União Consensual' (83 casos - 30,1\%), 'Casado' (57 casos - 20,7\%), 'Viúvo' (39 caos - 14,1\%), 'Separado Judicialmente' (8 casos - 2,9\%), respectivamente.

No que consiste a análise do tipo histológico, houve predominância do tipo 'Carcinoma Escamocelular, Soe', com registro de 200 casos, sendo 72,5\%. Então, fora seguido dos tipos 'Carcinoma Escamoso Ceratinizado, Soe' (23 casos - 8,3\%); 'Adenocarcinoma, Soe' (13 registros - 4,7\%); 'Adenocarcinoma Viloso' (08 casos - 2,9\%); 'Carcinoma "In Situ, Soe' (04 casos - 1,4\%) e do 'Carcinoma Soe' e 'Neoplasia IntraEpitelial Escamosa, Grau III' (ambos com 06 casos - 2,2\%), sendo estes s 6 tipos maior taxa em relação aos demais, constituindo-se em $92 \%$. Vale ressaltar que os tipos histológicos de menor registros foram os 'Adenocarcinoma Papilar, Soe' e o 'Carcinoma Escamoso, Micro-Invasor (C53.-)' com (1 caso - 0,4\%). Destes, observou-se, no que consiste a análise Descritiva, a Média de 2,3; sendo a Mediana com valor de 1,0. Além disso, o seu Desvio Padrão foi de 2,7; possuindo a Assimetria de 2,3 e a Variância de Amostra de 7,3 .

$\mathrm{Na}$ análise correspondente as Lesões de Caráter Neoplásico, houve registro de maior frequência no grupo de 'NIC III Displasia Acentuada' (70 casos - 25,4\%), seguido da 'NICI II Displasia Moderada' (60 casos $21,7 \%$ ), 'NIC I - Displasia Leve' (47 casos - 17,0\%), 'Carcinoma Escamocelular, SOE' (37 casos - 13,4\%), os quais comportaram $77,5 \%$ dos registros. Sendo que o tipo 'Carcinoma Sem HPV' fora registrado com 21 casos (7,6\%). Seguido da 'Cervicite Crônica'(15 casos - 5,4 \%). Sendo que os registrados 'Adenocarcinoma Epidermoide Invasivo', 'Adenocarcinoma Mucinoso', 'Carcinoma Escamocelular "In Situ" Com Invasão Questionável Do Estroma' corresponderam a 03 casos, 01 caso cada, com 1,2\%. No que consiste a análise Descritiva uma Média de 3,7, sendo a Mediana e o Modo, ambos, com 3,0. Sendo destes, o Desvio Padrão com 2,6 e a Assimetria de 1,3 .

Tabela 2 - Lesões de Caráter neoplásico.

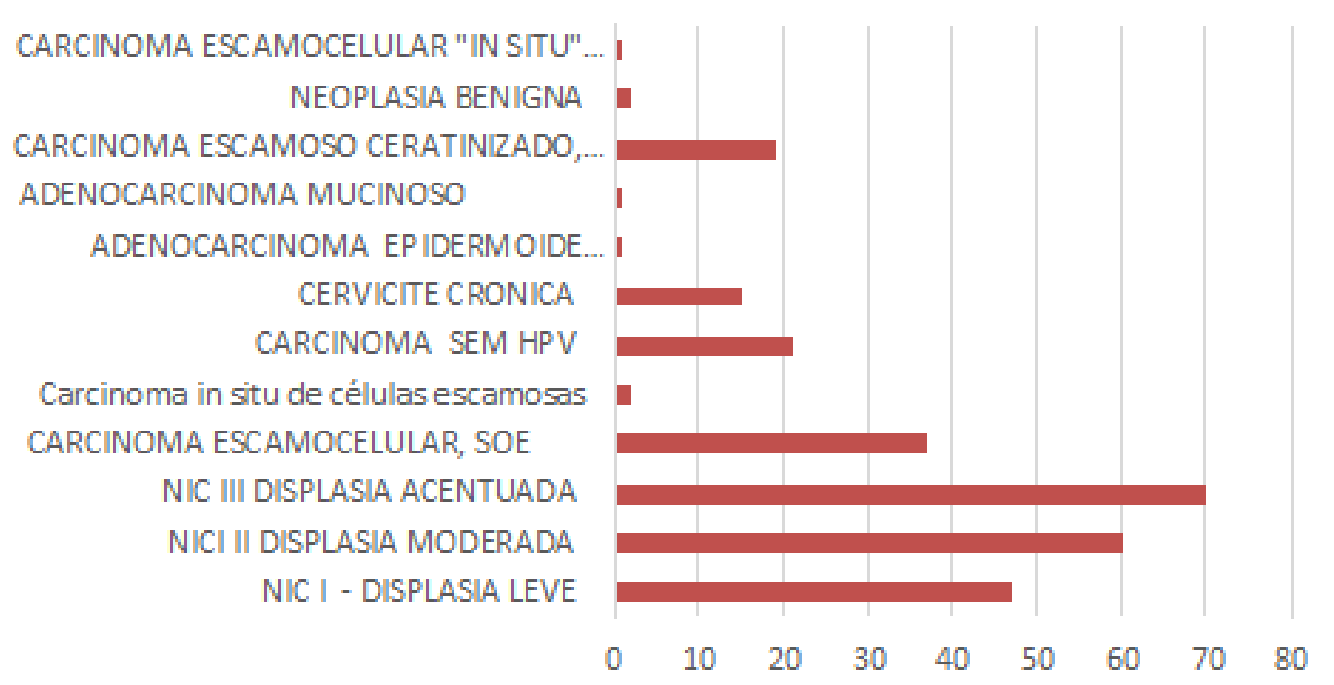

Fonte: SOARES NM, et al., 2020. 
Em relação a presença de HPV, observou-se no período registrado, que houve maior taxa de 'presença', com $68 \%$ (187 casos registrados), contrapartida a 32\% 'ausentes', com 89 casos.

No que consiste a Relação Pacientes Com HPV e o Histórico Familiar, observou-se registrado 138 casos (50\%) com a 'presença' de 'histórico familiar', sendo destes, 55\% (89 casos) com presença de HPV, sendo $48 \%$ (49 registros). Vale ressaltar que $38 \%$ (105 registrados) pertencem ao grupo que 'Não' possui 'Histórico Familiar', sendo que destes houve predominância do grupo que pertence ao com 'presença' da 'presença de HPV' (76 casos - $41 \%)$. Em contrapartida, 33 casos foram registrados como 'Sem Informação' (12\%).

Tabela 3 - Relação de pacientes com HPV e histórico familiar.

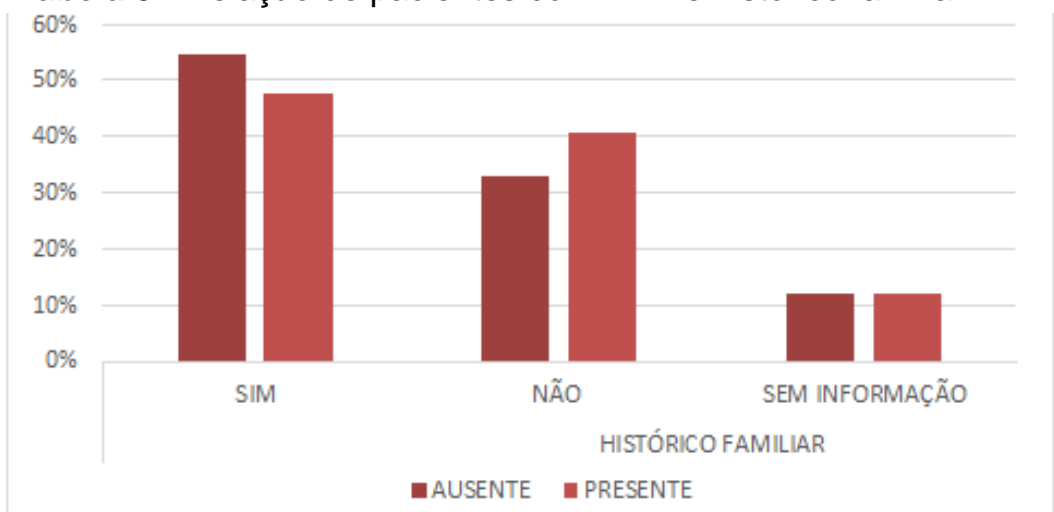

Fonte: SOARES NM, et al., 2020.

No que consiste na análise da relação escolaridade e o HPV, evidenciou-se naqueles os quais possuíam a 'presença' do HPV, o maior índice correspondente a 100\% dos casos aos que possuíam 'Nível Superior Incompleto' e, sendo seguido do grupo dos 'Analfabetos' (80\%), e dos grupos 'Fundamental Completo' e 'Nível Médio', ambos com $67 \%$. Em contrapartida, na relação dos registros de 'ausência do HPV', evidenciou-se a maior taxa no grupo de 'Nível Superior Completo' (40\%), seguida do 'Nível Fundamental Incompleto' (36\%) e dos grupos 'Fundamental Completo' e 'Nível Médio', ambos com 33\%.

Em relação ao estado conjugal e o HPV, na análise da 'presença' do HPV computou-se $67,75 \%$ (187 casos), sendo destes a maior taxa pertencente ao grupo 'Solteiro', com 61 registros $(22,10 \%)$, sendo seguido do grupo 'união consensual' com 52 casos (18,84\%) e dos casados, com 40 registros $(14,49 \%)$, sendo o grupo dos 'Viúvos' com 28 registrados $(10,14 \%)$ e os 'separados judicialmente' com 05 casos $(1,81 \%)$. Nesse contexto, 32,25\% (89 registros) correspondeu a 'ausência' de HPV, sendo destes, grande parte pertencente a 'União Consensual', com 31 casos (11,23\%), seguido dos 'solteiros' (26 registros -9,42\%) e dos 'casados' (17 registrados $-6,16 \%)$ e dos 'viúvos' com 11 casos $(3,99 \%)$, respectivamente.

Tabela 4 - Relação Estado Conjugal e a presença de HPV.

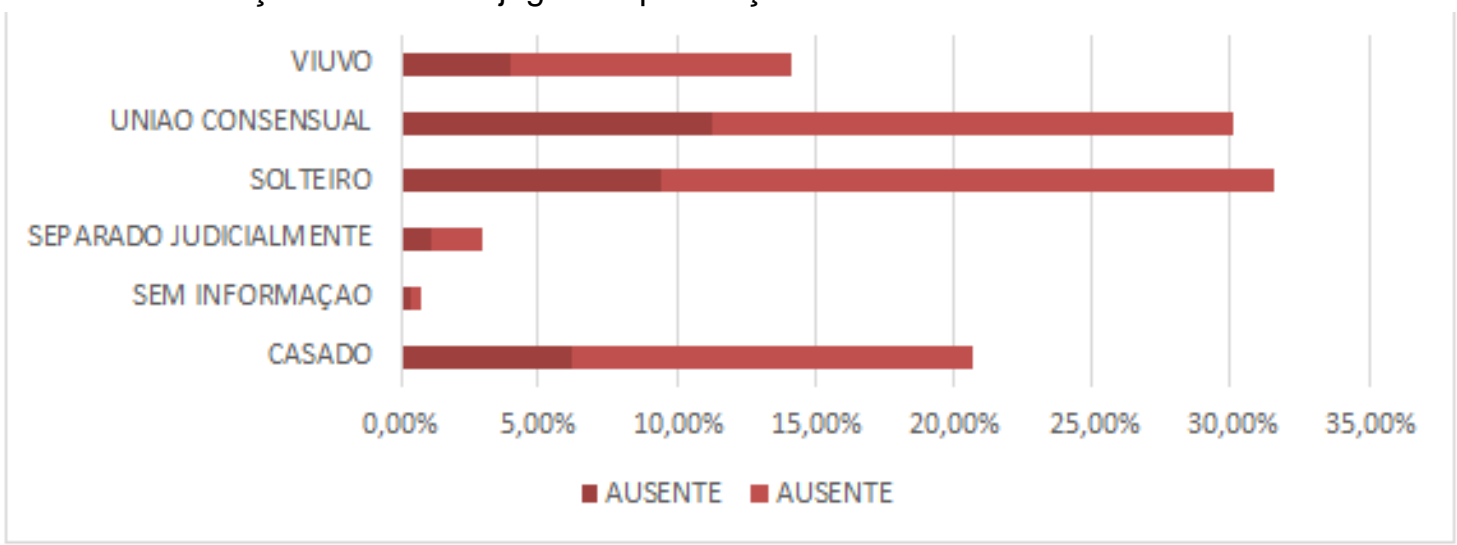

Fonte: SOARES NM, et al., 2020. 
Na correlação da infeç̧ão por HPV como fator de risco do câncer do colo do útero, observou-se que 32\% correspondia ao macro grupo de 'ausência' da 'presença de HPV' que as Lesões Neoplásicas 'Adenocarcinoma Epidermóide Invasivo' e 'Carcinoma Escamocelular "In Situ" Com Invasão Questionável Do Estroma' possuíram 100\%, a maior frequência, seguido do 'Carcinoma In Situ De Células Escamosas' e das 'Neoplasia Benigna', ambos com $50 \%$, com 'NIC I - Displasia Leve' com 38\%, sendo o 'NIC III Displasia Acentuada 'com 34\%, com o 'Carcinoma Sem HPV' com 33\% e 'Carcinoma Escamocelular, SOE' com 32\%.

Todavia, 68\% correspondem ao grupo de 'presença' da 'presença de HPV', sendo 'Adenocarcinoma Mucinoso' com 100\% de frequência, seguido da 'Cervicite Cronica' $(80 \%)$, do 'Carcinoma Escamoso Ceratinizado, SOE' (74\%), do 'NICI II Displasia Moderada' (73\%), do 'Carcinoma Escamocelular, SOE' (68\%), do 'Carcinoma Sem HPV' (67\%), 'Carcinoma in situ de células escamosas' e 'NEOPLASIA BENIGNA' (ambas com $50 \%$ ). No que consiste na análise descritiva dos dados registrados obtidos, observou-se a Mediana de 7,41; com a Variância de 66,08. Além disso, o intervalo de confiança entre as médias (IC 95\% (Dif. entre médias) fora de -20.23 a 3.89 .

\section{DISCUSSÃO}

No presente estudo foi encontrado uma predominância da faixa etária entre 40-60 anos, sendo um fator que corrobora diversos estudos que analisam a variação de idade entre mulheres com câncer de colo de útero pelo Brasil evidenciado por exemplo por Thuler LCS, et al. (2014) uma faixa etária média de 49,2 anos em uma análise nacional e Silva S, et al. (2018) aumentando esse valor de prevalência para mulheres até 65 anos de idade.

Dessa forma, a prevalência de casos de câncer de colo de útero estar nessa faixa etária corrobora a necessidade de rastreio dessa patologia em mulheres acima de 50 anos que, em geral, associam as patologias do canal cervical ou colo de útero (vulvovaginites e câncer de colo de útero) com atividade sexual, fazendo ter baixa adesão dessa faixa etária no exame, devendo ser um contexto que necessita ser combatido principalmente na atenção básica com educação em saúde realizado pela equipe profissional de saúde, estimulando as mulheres acima de 50 anos a realizar o exame de PCCU, principal exame de rastreio para câncer de colo de útero (SILVA S, et al., 2018)

Além disso, analisando ainda fatores sócios demográficos, é importante analisar a necessidade de realização do exame de PCCU para a faixa etária abaixo dos 40 anos, pois apesar da prevalência de câncer de colo de útero associado ao HPV ser entre 40-60 anos, estudos comprovam que alguns tipos de câncer iniciam com 30-35 anos e se manifestam aos 40 anos além de que o exame possui diversos benefícios implícitos em sua realização (BARBOSA IR, et al., 2017)

Quando a jovem vai ao encontro do exame de PCCU (Colpocitologia Oncótica), é identificado diversos fatores de risco na anamnese e exame físico geral que direcionam para o contato com o HPV e outas IST's (Infecções Sexualmente Transmissíveis), além da realização da inspeção prévia do canal cervical, identificando sinais sugestivos de malignidade do HPV e patologias que não se correlacionam diretamente com o câncer de colo de útero, mas também trazem desconforto na região pélvica, dores, dispareunia e pioram a qualidade de vida da mulher sendo um contexto que possui, melhorias na estrutura física, logística e profissional da atenção básica para acolher e realizar o exame de colpocitologia oncótica, educação em saúde efetiva sobre a temática e maior cobertura vacinal para o HPV, como medidas eficientes para prevenir ou identificar precocemente o câncer de colo de útero, seja associado ao vírus ou não (DAMACENA AM, et al., 2017).

As outras características dos aspectos sóciodemográficos como situação ocupacional e estado conjugal encontrados na presente pesquisa são divergentes quando comparado com a literatura atual associando a possibilidade de que em cada região do país há um perfil constante de idade da prevalência do câncer de colo de útero associado ao HPV, contudo, divergente quanto à outros aspectos estudados, sugerindo que idade e o nível de escolaridade são o fator preponderante quando comparado com os outros para ter câncer de colo de útero ou contato com o vírus HPV (DUARTE DV, et al., 2017). 
A análise de hábitos de vida de pacientes na atual pesquisa (49\% das mulheres) é, quando comparado com a literatura dessa temática, concordante em correlacionar o tabagismo como um dos principais fatores de risco para o desenvolvimento de câncer de colo de útero e outros de tipos de câncer que metastatizam para o útero, devendo ser um fator de risco combatido principalmente na atenção básica, propondo a utilização de terapia cognitiva comportamental, terapia de reposição de nicotina e medicações que possam auxiliar na cessação do tabagismo (BRITO-SILVA K, et al., 2014).

Observou-se predominância do tipo histológico Carcinoma Escamocelular com $72 \%$, seguido de Carcinoma Escamoso (8,3\%) e Adenocarcinoma (4,7\%) como os mais prevalentes na população estudada na atual pesquisa, bem como a análise correspondente as lesões de caráter neoplásico com maior frequência de NIC III Displasia Acentuada seguido de NIC II Displasia Moderada. Discordando desses dados, Silva S, et al. (2018) encontrou maior número de casos de pacientes, $86 \%$ e $85 \%$ respectivamente, com o tipo histológico Carcinoma Escamoso, e corroborando ZHOU et al (2017) que quando ao tipo mais prevalente de lesões de caráter neoplásico, levando em consideração a análise de que diferentes grupos de pacientes estudadas levarão à resultados de prevalência de histologia diferentes, sendo necessário, independente da prevalência com a população do local, fortalecimento da educação em saúde para conscientização da população em realização do exame de PCCU.

Aspectos pesquisados em relação ao vírus HPV avaliando as pacientes da coleta de dados analisam a presença do vírus em $68 \%$ das mulheres e relação de $50 \%$ de mulheres com histórico familiar de presença do HPV como contexto comum na vida das pacientes, corroborando com a literatura atual e evidenciando a necessidade de busca ativa das mulheres na família que possuem esse histórico e diminuição de fatores de risco nas que não possuem contato o HPV (SILVA S, et al., 2014).

Avalia-se a correlação do vírus com o grau de escolaridade, encontrando possível correlação de presença do HPV em mais de $80 \%$ dos casos de pacientes que tinham nível superior incompleto ou eram analfabetas, corroborando a avaliação inversamente proporcional de quanto maior a escolaridade, menor a chance de contato com o vírus (AYRES ARG, et al., 2013).

\section{CONCLUSÃO}

Logo, conclui-se que há fatores que influenciam o diagnóstico tardio e a negligência de sinais e sintomas preditivos de câncer de colo de útero tais como baixo nível de escolaridade, idade de prevalência, tabagismo e principalmente contato HPV. Além disso, foi possível avaliar a prevalência dos tipos histológicos de câncer de colo de útero associado à infecção do HPV em pacientes da pesquisa. Apesar disso, são necessários mais estudos nessa temática a fim de agilizar o manejo e tratamento das pacientes com diagnóstico de CA de colo de útero e atuar na educação em saúde para conscientizar a população feminina sobre o benefício do exame de preventivo.

\section{REFERÊNCIAS}

1. AYRES ARG, et al. Tendência da incidência de câncer do colo do útero invasor em quatro capitais brasileiras: dados dos registros de câncer de base populacional, 1990-2004. Cad. saúde colet., Rio de Janeiro, 2013, 21(3): $289-295$.

2. BALBI FSM, et al. Estudo da prevalência da infecção pelo Papiloma Vírus Humano (HPV) em mulheres no climatério em um hospital de referência de Belém. 2015. Dissertação (Mestrado) - Universidade Federal do Pará, Núcleo de Medicina Tropical, Belém, 2015. Programa de Pós-Graduação em Doenças Tropicais.

3. BARBOSA IR. Regional and Socioeconomic Differences in the Coverage of the Papanicolau Test in Brazil: Data from the Brazilian Health Survey 2013. Rev. Bras. Ginecol. Obstet., 2017, 39(9): 480-487.

4. BRASIL. Ministério da Saúde. Federação Brasileira das Associações de Ginecologia e Obstetrícia. Rastreamento para Câncer de Colo Uterino o que há de novo. Ceará, 2017.

5. BRASIL. Ministério da Saúde. Instituto Nacional do Câncer. HPV e Câncer- Perguntas mais frequente. Rio de Janeiro: Ministério da Saúde, 2016.

6. BRASIL. Ministério da Saúde. Instituto Nacional do Câncer. Infecção e Câncer. Rio de Janeiro: Ministério da Saúde, 2016.

7. BRASIL. Ministério da Saúde. Instituto Nacional do Câncer. Tipos de câncer: Colo do Útero. Rio de Janeiro: Ministério da Saúde, 2016. 
8. BRITO-SILVA K, et al. Integralidade no cuidado ao câncer do colo do útero: avaliação do acesso. Rev. Saúde Pública, 2014, 48(2): 240-248.

9. CARVALHO MC, et al. Mulheres portadoras de lesões precursoras do câncer do colo do útero e HPV: descrição do perfil socioeconômico e demográfico. J Bras Doenças Sex Transm.; 2011, 23(1): 28-33

10. CASARIN MR. Educação em saúde para prevenção do câncer de colo do útero em mulheres do município de Santo Ângelo/RS. Ciência \& Saúde Coletiva, 2011, 16: 3925-3932.

11. DAMACENA AM. Rastreamento do câncer do colo do útero em Teresina, Piauí: estudo avaliativo dos dados do Sistema de Informação do Câncer do Colo do Útero, 2006-2013. Epidemiol. Serv. Saúde, 2017, 26(1): 71-80.

12. DE SOUZA AF, et al. Conhecimento de mulheres sobre HPV e câncer do colo do útero após consulta de enfermagem. Revista Brasileira de Cancerologia, 2015, 61(4): 343-350.

13. DUARTE DV, et al. Prevalence of human Papillomavirus Infection and Cervical Cancer screening among Riverside women of the Brazilian Amazon. Rev. Bras. Ginecol. Obstet. 2017, 39(7):10-20,

14. SOUSA ACO, et al. Caracterização das Alterações Citopatológicas e Fatores de Riscos Associados ao Desenvolvimento do Câncer de Colo Útero. Revista UningáReview, 2018, 30(1):37-42.

15. MACHADO HS, et al. Câncer de colo de útero: análise Epidemiológica e Citopatológica no município de VassourasRJ. Revista Pró-UniverSUS, 2017, 8(1): 23-34.

16. SILVA DSM, et al. Rastreamento do câncer do colo do útero no Estado do Maranhão, Brasil. Ciênc. saúde coletiva, 2014, 19(4): 1163-1170.

17. SILVA RCG, et al. Perfil de mulheres com câncer de colo do útero atendidas para tratamento em centro de oncologia. Rev. Bras. Saude Mater. Infant., 2018, 18(4): 695-702.

18. TEIXEIRA JC, et al. Particularização do adenocarcinoma do colo frente ao conhecimentoatual. Femina,, 2012, 40(5): 270-283.

19. THULER LCS, et al. Determinantes do diagnóstico em estadio avançado do câncer do colo do útero no Brasil. Rev. Bras. Ginecol. Obstet., 2014, 36(6): 237-243.

20. ZHOU J, et al. Comparison of clinical outcomes of squamous cell carci-noma, adenocarcinoma, and adenosquamous carcinoma of the uterine cervix after definitive radiotherapy: a population-based analysis. J Cancer Res Clin Oncol.; 2017, 143(1): 115-22. 